\title{
Screening Technique for Identification of Resistant Genotypes against Post Flowering Stalk Rot Complex caused by Macrophomina phaseolina in Maize (Zea mays L.)
}

\author{
Banoth Madhu*, K. Prabhavathi, D. Bhadru and B. Mallaiah \\ Department of Genetics and Plant Breeding, College of Agriculture, Rajendranagar, \\ Professor Jayashankar Telangana State Agricultural University, Hyderabad-500 030, India \\ *Corresponding author
}

\section{A B S T R A C T}

\begin{tabular}{|l|}
\hline Ke y w o r d s \\
Maize genotypes, \\
$\begin{array}{l}\text { Lines, Testers, } \\
\text { Crosses, Standard } \\
\text { checks, Tooth pick } \\
\text { method of } \\
\text { inoculation, Rating } \\
\text { scale, Resistant, } \\
\text { Screening, Disease } \\
\text { score, Stalk rot } \\
\text { complex }\end{array}$ \\
\hline $\begin{array}{l}\text { Article Info } \\
\text { Accepted: } \\
\text { 20 December } 2020 \\
\text { Available Online: } \\
\text { 10 January } 2021\end{array}$ \\
\hline
\end{tabular}

Keywords

Maize genotypes,

Lines, Testers,

checks, Tooth pick

method of

scale, Resistant,

Screening, Disease

score, Stalk rot

In order to screen maize genotypes for resistance to post-flowering stalk rot (PFSR) complex caused by Macrophomina phaseolina under field conditions, toothpick method was used for creating artificial epiphytotics. In this study, 98 maize genotypes (20 parents (15 Lines $\times 5$ testers), 75 Single Cross Hybrids (SCHs) and three standard checks) were screened in field by toothpick method of inoculation. The field screening of maize genotypes by the standard toothpick method which needs about 40 days (only at harvesting stage) for expression of plant drying symptoms due to PFSR and data are possible to record only at the time of crop harvesting. Screening is done by using 1-9 rating scale of PFSR for scoring disease severity in-vivo condition. All these maize inbred lines were screened in field by toothpick method of inoculation at Maize Research Centre, Agricultural Research Institute, Rajendranagar, Hyderabad. As a result, most of the genotypes were exhibited disease reaction varying from resistant (score-2) to moderately resistant (score-5) against $M$. phaseolina. In ordered to identified PFSR resistant lines, screening of 98 maize genotypes in field against $M$. phaseolina, only four lines, viz., MGC-237, MGC-248, MGC-254, MGC-256 and two testers, viz., BML-6 and GP-311. Whereas, 15 crosses viz., MGC-9 $\times$ BML-6, MGC-9 $\times$ BML-14, MGC-32 × BML-14, MGC-32 × GP-170, MGC-92 × GP-170, MGC-137 × GP-311, MGC-237 × BML-7, MGC-242 × BML-14, MGC-248 × GP-311, MGC-252 × BML-14, MGC-252 × GP-311, MGC-254 $\times$ BML-14, MGC-254 $\times$ GP-311, MGC-256 $\times$ GP-170 and MGC-256 $\times$ GP-311 were found resistant.

\section{Introduction}

Maize (Zea mays L. $2 \mathrm{n}=2 \mathrm{x}=20$ ) is known as Miracle crop and Queen of cereals because of its highest genetic yield potential among the cereals. The global maize production is about 1.09 billion metric tonnes from 153.0 million hectares. The USA has highest productivity (10.57 $\mathrm{t} \mathrm{ha}^{-1}$ ), which is double than the global average $\left(4.92 \mathrm{t} \mathrm{ha}^{-1}\right)$. Whereas, the average productivity in India is about 2.68 $\mathrm{t} \mathrm{ha}^{-1}$ with production of 24.26 million tonnes from 9.3 million hectares, the country lags far behind in productivity against world average. 
However, in Telangana State maize is grown in almost all the districts in an area of 0.64 million hectares, with a production of about 2.60 million tonnes (INDIASTAT, 2016-17).

In India yield lag is one of the major constraints that hinder maize production. Apart from pest and diseases, fungal diseases like, post flowering stalk rots (PFSR) poses a major threat to the productivity of maize (Sharma et al., 1993). PFSR is a complex disease of maize, which commonly appears when there is scarcity of irrigation coupled with high soil temperature at flowering stage of the crop. PFSR is caused by different fungal pathogens but, Charcoal rot by Macrophomina phaseolina is more prevalent and destructive in Telangana State as well as in Rajasthan, Bihar, Andhra Pradesh, Uttar Pradesh, Punjab, Madhya Pradesh and West Bengal. Stalk rot is found to be prevalent in the plains only in the kharif crop when summer temperature becomes relatively high $\left(30^{\circ}\right.$ to $\left.35^{\circ} \mathrm{C}\right)$. The disease incidence, recorded in India time to time, ranged from 10.0 to $42.0 \%$ (Desai et al., 1991), 13.2 to $39.5 \%$ (Payak and Sharma 1985), 25.0 to $32.0 \%$ (Kumar et al., 1998), 10.0 to $42.0 \%$ (Harlapur et al., 2002), 25.0 to $32.2 \%$ (Krishna et al., 2013) and in recent years yield reduction has been reported to be as high as 22.3 to $63.5 \%$ (AICRP, 2014).

In order to combat this problem, development of maize cultivars with genetic resistant represent one of the most cost-efficient, safe and eco-friendly solutions for reducing the yield losses caused by PFSR compared to chemical and biological control methods (Nagy and Cabulea, 1996). Information on the nature of inheritance of PFSR resistance is lacking, which is a prerequisite to initiate appropriate breeding program for the development of PRSR resistant varieties, on which very little emphasis had been made so far. To develop disease resistant varieties, screening of available genotypes against the pathogens was done under artificial epiphytotic condition and it yielded a set of stalk rot resistant germplasm in India (Shekhar et al., 2010, Hooda et al., 2012) and abroad (Clark and Foley 1985). In India, artificial epiphytotic condition for stalk rot disease is created by inoculating the plants in the field just after flowering mainly by toothpick method of inoculation (Anon. 1983, 2012). But this method requires longer time for disease development and rotting symptoms in the inoculated stalks become prominent only at harvesting stage. But now, in the current investigation tooth pick method is used in the field.

\section{Materials and Methods}

Disease sick plot development is crucial in successes of breeding for resistance to PFSR. Screening reinforced with artificial inoculation using tooth pick method is effective in supplementing the disease sick plot technique of screening against PFSR. The methodology fallowed is suitable for screening against a multi-pathogen disease complex (Shankar Lingam and Venkatesh, 2005). Post-flowering stalk rot of maize occur in both the growing season's viz., kharif (rainy) and Rabi (winter) at Maize Research Centre, Agriculture Research Institute, Rajendranagar, Hyderabad, a disease sick plot was developed by incorporating infected stubbles of all the casual organisms of PFSR over a period of more than three decades.

Artificial inoculation was done with tooth picks on which the disease casual organisms were grown in the laboratory. For this purpose, infected maize stems with PFSR were collected, cut into small bits and surface sterilized with $0.1 \%$ mercuric chloride for one minute followed by washing with sterile distilled water. Finally, a single bit was aseptically transferred to sterilized $10 \mathrm{~cm}$ 
Petri plates containing $20 \mathrm{ml}$ of sterilized Potato Dextrose Agar medium (PDA). The plates were incubated for three days at $24 \pm$ 20c. The fungal hyphae were then aseptically transferred to culture tubes containing the sterile PDA medium and incubated for 10 days to get the stock culture of the pathogen. $100 \mathrm{ml}$ (peptone 1g, honey $5 \mathrm{ml}$ and distilled water $94 \mathrm{ml})$ sterilized (20 minutes) and cooled honey peptone medium was poured under aseptic condition into a sterilized, wide mouthed bottle with screw cap, containing toothpicks.

Then from stock culture, two loops of mycelial suspension were seeded in bottle containing toothpicks under aseptic conditions. Then bottles were incubated at 350c for 7 days. The toothpicks covered with abundant mycelia of the fungus were then ready to use in about 10 days in field inoculation.

Seeds of 20 maize genotypes were collected from the Maize Research Centre (MRC), Hyderabad. The genotypes were crossed to generate 75 SCHs during Kharif, 2019 in L $\times$ $\mathrm{T}$ mating design (Table 1) and these 20 parents (15 Lines and 5 Testers) and $75 \mathrm{SCHs}$ along with three standard checks, a total of 98 genotypes were subjected to evaluated by raising the crop in disease sick plot accompanied by toothpick inoculation during Rabi, 2019-20, at the same institute.

Inoculation of the plants of 45-50 days old was done just after flowering by toothpick method (Anon. 1983 and 2012. Before inoculation, one jabber was made by driving/fixing a nail of toothpick size into a wooden handle. For inoculation, most appropriate plant stage for inoculation is between tasseling and pollination for that the lower internode (second or third) above soil level was selected. Then the pointed head of the nail was pushed carefully into the selected internode to make a hole of desired length $(2 \mathrm{~cm})$. The round toothpick bearing inoculums were inserted into the hole that effectively sealed the hole to prevent drying of the inoculums.

Typical symptoms like partial or whole plant drying appear in the inoculated plants about 20-25 days post-inoculation (DPI). Classification for the reactions for the pathogens was done on an individual plant basis, splitting the stalk open and observing the rot is the most reliable method of determining the amount and extent of stalk rot and the 1-9 index scale, suggested by Payak and Sharma (1983) was followed for scoring and scale has been unequally distributed into four categories of disease severity (Table 2), viz., resistant (R), moderately resistant (MR), moderately susceptible (MS) and susceptible reaction $(\mathrm{S})$.

All data on the disease severity generated from the experiments conducted in field was assessed at the end.

\section{Results and Discussion}

Screening of maize genotypes in field (Table 3): Out of the 15 lines screened against $M$. phaseolina, only four lines, viz., MGC-237, MGC-248, MGC-254 and MGC-256 were found resistant, four lines, viz., MGC-9, MGC-137, MGC-242 and MGC-252 were moderately resistant, five lines, viz., MGC-6, MGC-32, MGC-92, MGC-238 and MGC-239 were moderately susceptible and only two lines, viz., MGC-15 and MGC-230 were found susceptible. Whereas, Out of the 5 testers screened against $M$. phaseolina, only two testers, viz., BML-6 and GP-311 were found resistant, one tester, GP-170 was moderately resistant, one tester, BML-14 was moderately susceptible and only one tester, BML-7 was found susceptible. 
Table1 List of maize inbred lines and crosses used for identification of PFSR resistant and susceptible genotypes

\begin{tabular}{|c|c|c|c|}
\hline S.No & Maize genotypes & S. No & Maize genotypes \\
\hline & Lines & & \\
\hline 1 & MGC-6 & 50 & MGC-137 × GP-311 \\
\hline 2 & MGC-9 & 51 & MGC-230 × BML-6 \\
\hline 3 & MGC-15 & 52 & MGC-230 × BML-7 \\
\hline 4 & MGC-32 & 53 & MGC-230 $\times$ BML-14 \\
\hline 5 & MGC-92 & 54 & MGC-230 × GP-170 \\
\hline 6 & MGC-137 & 55 & MGC-230 $\times$ GP-311 \\
\hline 7 & MGC-230 & 56 & MGC-237 × BML-6 \\
\hline 8 & MGC-237 & 57 & MGC-237 × BML-7 \\
\hline 9 & MGC-238 & 58 & MGC- $237 \times$ BML-14 \\
\hline 10 & MGC-239 & 59 & MGC-237 × GP-170 \\
\hline 11 & MGC-242 & 60 & MGC-237 × GP-311 \\
\hline 12 & MGC-248 & 61 & MGC- $238 \times$ BML- 6 \\
\hline 13 & MGC-252 & 62 & MGC-238 × BML-7 \\
\hline 14 & MGC-254 & 63 & MGC-238 $\times$ BML-14 \\
\hline \multirow[t]{2}{*}{15} & MGC-256 & 64 & MGC-238 × GP-170 \\
\hline & Testers & 65 & MGC-238 $\times$ GP-311 \\
\hline 16 & BML-6 & 66 & MGC-239 × BML-6 \\
\hline 17 & BML-7 & 67 & MGC-239 × BML-7 \\
\hline 18 & BML-14 & 68 & MGC-239 × BML-14 \\
\hline 19 & GP-170 & 69 & MGC-239 × GP-170 \\
\hline \multirow[t]{2}{*}{20} & GP-311 & 70 & MGC-239 × GP-311 \\
\hline & Crosses & 71 & MGC-242 × BML-6 \\
\hline 21 & MGC- $6 \times$ BML-6 & 72 & MGC-242 × BML-7 \\
\hline 22 & MGC-6 × BML-7 & 73 & MGC-242 × BML-14 \\
\hline 23 & MGC- $6 \times$ BML-14 & 74 & MGC- $242 \times$ GP-170 \\
\hline 24 & MGC- $6 \times$ GP-170 & 75 & MGC-242 $\times$ GP-311 \\
\hline 25 & MGC- $6 \times$ GP-311 & 76 & MGC-248 × BML-6 \\
\hline 26 & MGC-9 $\times$ BML-6 & 77 & MGC- $248 \times$ BML-7 \\
\hline 27 & MGC-9 × BML-7 & 78 & MGC-248 $\times$ BML-14 \\
\hline 28 & MGC-9 $\times$ BML-14 & 79 & MGC-248 $\times$ GP-170 \\
\hline 29 & MGC-9 $\times$ GP-170 & 80 & MGC-248 $\times$ GP-311 \\
\hline 30 & MGC- $9 \times$ GP-311 & 81 & MGC-252 × BML-6 \\
\hline 31 & MGC-15 × BML-6 & 82 & MGC-252 × BML-7 \\
\hline 32 & MGC-15 × BML-7 & 83 & MGC-252 × BML-14 \\
\hline 33 & MGC-15 × BML-14 & 84 & MGC- $252 \times$ BML-170 \\
\hline 34 & MGC-15 $\times$ GP-170 & 85 & MGC- $252 \times$ BML-311 \\
\hline 35 & MGC-15 × GP-311 & 86 & MGC-254 × BML-6 \\
\hline 36 & MGC-32 × BML-6 & 87 & MGC-254 × BML-7 \\
\hline 37 & MGC-32 × BML-7 & 88 & MGC-254 × BML-14 \\
\hline 38 & MGC-32 × BML-14 & 89 & MGC-254 × GP-170 \\
\hline 39 & MGC-32 $\times$ GP-170 & 90 & MGC-254 × GP-311 \\
\hline 40 & MGC-32 $\times$ GP-311 & 91 & MGC-256 × BML-6 \\
\hline 41 & MGC-92 × BML-6 & 92 & MGC-256 × BML-7 \\
\hline 42 & MGC-92 × BML-7 & 93 & MGC-256 $\times$ BML-14 \\
\hline 43 & MGC-92 × BML-14 & 94 & MGC-256 × GP-170 \\
\hline 44 & MGC-92 9 GP-170 & 95 & MGC-256 $\times$ GP-311 \\
\hline 45 & MGC-92 $\times$ GP-311 & & Checks \\
\hline 46 & MGC-137 × BML-6 & 96 & DHM-117 \\
\hline 47 & MGC-137 × BML-7 & 97 & BIO-9544 \\
\hline 48 & MGC-137 × BML-14 & 98 & KAVERI-50 \\
\hline 49 & MGC-137 × GP-170 & & \\
\hline
\end{tabular}


Table.2 Disease rating scale for scoring disease severity of PFSR

\begin{tabular}{|c|c|c|}
\hline Disease rating scale & Disease severity percentage (\%) & Disease reaction \\
\hline 1 & $\begin{array}{l}\text { Healthy or trace/slight discolouration at the } \\
\text { site of inoculation }\end{array}$ & Immune reaction \\
\hline 2 & $\begin{array}{l}\text { Up to } 50 \% \text { of the inoculated internode is } \\
\text { discoloured }\end{array}$ & \multirow[t]{2}{*}{$\begin{array}{l}\text { Resistant } \\
(\text { Score: } \leq 3.0)\end{array}$} \\
\hline 3 & $\begin{array}{l}51-75 \% \text { of the inoculated internode is } \\
\text { discoloured }\end{array}$ & \\
\hline 4 & $\begin{array}{l}\text { 76-100\% of the inoculated resistant } \\
\text { internode is discoloured }\end{array}$ & \multirow[t]{2}{*}{$\begin{array}{l}\text { Moderately resistant } \\
\text { (Score: } 3.1-5.0)\end{array}$} \\
\hline 5 & $\begin{array}{l}\text { Less than } 50 \% \text { discolouration of the } \\
\text { adjacent internode }\end{array}$ & \\
\hline 6 & $\begin{array}{l}\text { More than } 50 \% \text { discolouration of the } \\
\text { adjacent internode }\end{array}$ & \multirow[t]{2}{*}{$\begin{array}{l}\text { Moderately susceptible } \\
\quad \text { (Score: } 5.1-7.0)\end{array}$} \\
\hline 7 & Discolouration of three internodes & \\
\hline 8 & Discolouration of four internodes & \multirow[t]{2}{*}{$\begin{array}{l}\text { Susceptible } \\
(\text { Score: } \geq 7.0)\end{array}$} \\
\hline 9 & $\begin{array}{l}\text { Discolouration of five or more internodes } \\
\text { and premature death of plant }\end{array}$ & \\
\hline
\end{tabular}

Table.3 Disease incidence of Macrophomina stalk rot recorded in field by toothpick method (at harvesting) using standard rating scale (score 1-9)

\begin{tabular}{|c|c|c|}
\hline \multirow{2}{*}{ Parent/Cross } & \multicolumn{2}{|c|}{ In field (Toothpick method) } \\
\cline { 2 - 3 } & $\begin{array}{c}\text { MP mean score } \\
\text { Lisease reaction }\end{array}$ \\
\hline & 7 & \\
\hline MGC-6 & 5 & MS \\
\hline MGC-9 & 8 & S \\
\hline MGC-15 & 7 & MS \\
\hline MGC-32 & 6 & MS \\
\hline MGC-92 & 4 & MR \\
\hline MGC-137 & 8 & S \\
\hline MGC-230 & 3 & R \\
\hline MGC-237 & 6 & MS \\
\hline MGC-238 & 7 & MS \\
\hline MGC-239 & 5 & MR \\
\hline MGC-242 & 5 & R \\
\hline MGC-248 & 3 & MR \\
\hline MGC-252 & 4 & R \\
\hline MGC-254 & 3 & R \\
\hline MGC-256 & 2 & \\
\hline
\end{tabular}




\begin{tabular}{|c|c|c|}
\hline Testers & & \\
\hline BML-6 & 3 & $\mathrm{R}$ \\
\hline BML-7 & 8 & $\mathrm{~S}$ \\
\hline BML-14 & 7 & MS \\
\hline GP-170 & 5 & MR \\
\hline GP-311 & 3 & $\mathrm{R}$ \\
\hline \multicolumn{3}{|c|}{ Crosses } \\
\hline MGC- $6 \times$ BML- 6 & 7 & MS \\
\hline MGC- $6 \times$ BML-7 & 5 & MR \\
\hline MGC-6 $\times$ BML-14 & 4 & MR \\
\hline MGC- $6 \times$ GP- 170 & 4 & MR \\
\hline MGC- $6 \times$ GP-311 & 6 & MS \\
\hline MGC-9 × BML-6 & 3 & $\mathrm{R}$ \\
\hline MGC-9 × BML-7 & 7 & MS \\
\hline MGC-9 $\times$ BML-14 & 3 & $\mathrm{R}$ \\
\hline MGC-9 $\times$ GP- 170 & 8 & $\mathrm{~S}$ \\
\hline MGC-9 × GP-311 & 7 & MS \\
\hline MGC-15 × BML-6 & 7 & MS \\
\hline MGC-15 × BML-7 & 8 & $\mathrm{~S}$ \\
\hline MGC-15 × BML-14 & 8 & $S$ \\
\hline MGC-15 × GP-170 & 8 & $\mathrm{~S}$ \\
\hline MGC-15 × GP-311 & 5 & MR \\
\hline MGC-32 × BML-6 & 4 & MR \\
\hline MGC-32 × BML-7 & 8 & $\mathrm{~S}$ \\
\hline MGC-32 × BML-14 & 2 & $\mathrm{R}$ \\
\hline MGC-32 × GP-170 & 3 & $\mathrm{R}$ \\
\hline MGC-32 × GP-311 & 4 & MR \\
\hline MGC-92 × BML-6 & 8 & $\mathrm{~S}$ \\
\hline MGC-92 × BML-7 & 6 & MS \\
\hline MGC-92 × BML-14 & 8 & $\mathrm{~S}$ \\
\hline MGC-92 × GP-170 & 3 & $\mathrm{R}$ \\
\hline MGC-92 × GP-311 & 6 & MS \\
\hline MGC-137 × BML-6 & 7 & MS \\
\hline MGC-137 × BML-7 & 8 & $\mathrm{~S}$ \\
\hline MGC-137 × BML-14 & 6 & MS \\
\hline MGC-137 × GP-170 & 4 & MR \\
\hline MGC-137 × GP-311 & 2 & $\mathrm{R}$ \\
\hline MGC-230 × BML-6 & 6 & MS \\
\hline MGC-230 × BML-7 & 4 & MR \\
\hline MGC-230 $\times$ BML-14 & 8 & $\mathrm{~S}$ \\
\hline MGC-230 × GP-170 & 8 & $\mathrm{~S}$ \\
\hline MGC-230 × GP-311 & 5 & MR \\
\hline MGC-237 × BML-6 & 8 & $\mathrm{~S}$ \\
\hline MGC-237 × BML-7 & 3 & $\mathrm{R}$ \\
\hline MGC-237 × BML-14 & 8 & $\mathrm{~S}$ \\
\hline
\end{tabular}




\begin{tabular}{|c|c|c|}
\hline MGC-237 × GP-170 & 7 & MS \\
\hline MGC-237 × GP-311 & 7 & MS \\
\hline MGC-238 × BML-6 & 8 & $\mathrm{~S}$ \\
\hline MGC-238 × BML-7 & 5 & MR \\
\hline MGC-238 $\times$ BML-14 & 6 & MS \\
\hline MGC-238 × GP-170 & 7 & MS \\
\hline MGC-238 × GP-311 & 9 & $\mathrm{~S}$ \\
\hline MGC-239 × BML-6 & 8 & S \\
\hline MGC-239 × BML-7 & 7 & MS \\
\hline MGC-239 × BML-14 & 4 & MR \\
\hline MGC-239 × GP-170 & 8 & $\mathrm{~S}$ \\
\hline MGC-239 × GP-311 & 5 & MR \\
\hline MGC-242 × BML-6 & 9 & $\mathrm{~S}$ \\
\hline MGC-242 × BML-7 & 7 & MS \\
\hline MGC-242 $\times$ BML-14 & 3 & $\mathrm{R}$ \\
\hline MGC-242 × GP-170 & 4 & MR \\
\hline MGC-242 × GP-311 & 6 & MS \\
\hline MGC-248 $\times$ BML-6 & 8 & $\mathrm{~S}$ \\
\hline MGC-248 × BML-7 & 7 & MS \\
\hline MGC-248 $\times$ BML-14 & 4 & MR \\
\hline MGC-248 $\times$ GP-170 & 7 & MS \\
\hline MGC-248 × GP-311 & 3 & $\mathrm{R}$ \\
\hline MGC-252 × BML-6 & 5 & MR \\
\hline MGC-252 $\times$ BML-7 & 8 & $\mathrm{~S}$ \\
\hline MGC-252 $\times$ BML-14 & 2 & $\mathrm{R}$ \\
\hline MGC-252 × GP-170 & 7 & MS \\
\hline MGC-252 $\times$ GP-311 & 3 & $\mathrm{R}$ \\
\hline MGC-254 × BML-6 & 9 & S \\
\hline MGC-254 × BML-7 & 8 & $S$ \\
\hline MGC-254 × BML-14 & 3 & $\mathrm{R}$ \\
\hline MGC-254 × GP-170 & 8 & $S$ \\
\hline MGC-254 × GP-311 & 3 & $\mathrm{R}$ \\
\hline MGC-256 × BML-6 & 7 & MS \\
\hline MGC-256 × BML-7 & 7 & MS \\
\hline MGC-256 × BML-14 & 4 & MR \\
\hline MGC-256 × GP-170 & 3 & $\mathrm{R}$ \\
\hline MGC-256 × GP-311 & 2 & $\mathrm{R}$ \\
\hline \multicolumn{3}{|c|}{ Checks } \\
\hline DHM-117 & 3 & $\mathrm{R}$ \\
\hline BIO-9544 & 5 & MR \\
\hline KAVERI-50 & 9 & $\mathrm{~S}$ \\
\hline
\end{tabular}

MP: Macrophomina phaseolina, R: Resistant, MR: Moderately resistant, MS: Moderately susceptible, S: Susceptible. 
Table.4 Summary of post-flowering stalk rot (PFSR) disease reaction observed in field condition

\begin{tabular}{|c|c|c|}
\hline \multirow{2}{*}{ Parent/Cross } & \multicolumn{2}{|c|}{ In field (Toothpick method) } \\
\hline & MP mean score & Disease reaction \\
\hline MGC-9 & 5 & MR \\
\hline MGC-137 & 4 & MR \\
\hline MGC-237 & 3 & $\mathrm{R}$ \\
\hline MGC-242 & 5 & MR \\
\hline MGC-248 & 3 & $\mathrm{R}$ \\
\hline MGC-252 & 4 & MR \\
\hline MGC-254 & 3 & $\mathrm{R}$ \\
\hline MGC-256 & 2 & $\mathrm{R}$ \\
\hline BML-6 & 3 & $\mathrm{R}$ \\
\hline GP-170 & 5 & MR \\
\hline GP-311 & 3 & $\mathrm{R}$ \\
\hline MGC- $6 \times$ BML-7 & 5 & MR \\
\hline MGC- $6 \times$ BML-14 & 4 & MR \\
\hline MGC- $6 \times$ GP- -170 & 4 & MR \\
\hline MGC-9 × BML-6 & 3 & $\mathrm{R}$ \\
\hline MGC-9 $\times$ BML-14 & 3 & $\mathrm{R}$ \\
\hline MGC-15 ×GP-311 & 5 & MR \\
\hline MGC-32 $\times$ BML- 6 & 4 & MR \\
\hline MGC-32 $\times$ BML-14 & 2 & $\mathrm{R}$ \\
\hline MGC-32 $\times$ GP-170 & 3 & $\mathrm{R}$ \\
\hline MGC-32 ×GP-311 & 4 & MR \\
\hline MGC- $92 \times$ GP-170 & 3 & $\mathrm{R}$ \\
\hline MGC-137 $\times$ GP-170 & 4 & MR \\
\hline MGC-137 ×GP-311 & 2 & $\mathrm{R}$ \\
\hline MGC-230 ×GP-311 & 5 & MR \\
\hline MGC-237 $\times$ BML-7 & 3 & $\mathrm{R}$ \\
\hline MGC-238 $\times$ BML-7 & 5 & MR \\
\hline MGC-239 $\times$ BML-14 & 4 & MR \\
\hline MGC-239 ×GP-311 & 5 & MR \\
\hline MGC- $242 \times$ BML-14 & 3 & $\mathrm{R}$ \\
\hline MGC-242 ×GP-170 & 4 & MR \\
\hline MGC-248 $\times$ BML-14 & 4 & MR \\
\hline MGC-248 ×GP-311 & 3 & $\mathrm{R}$ \\
\hline MGC-252 × BML-6 & 5 & MR \\
\hline MGC-252 $\times$ BML-14 & 3 & $\mathrm{R}$ \\
\hline MGC-252 $\times$ GP-311 & 3 & $\mathrm{R}$ \\
\hline MGC-254 × BML-14 & 3 & $\mathrm{R}$ \\
\hline MGC-254 × GP-311 & 3 & $\mathrm{R}$ \\
\hline MGC-256 $\times$ BML-14 & 4 & MR \\
\hline MGC-256 $\times$ GP-170 & 3 & $\mathrm{R}$ \\
\hline MGC-256 ×GP-311 & 2 & $\mathrm{R}$ \\
\hline DHM-117 & 3 & $\mathrm{R}$ \\
\hline BIO-9544 & 5 & MR \\
\hline
\end{tabular}

MP: Macrophomina phaseolina, R: Resistant, MR: Moderately resistant, MS: Moderately susceptible, S: Susceptible. 
Whereas, among the 75 SCHs hybrids, 15 hybrids viz., MGC-9 × BML-6, MGC-9 $\times$ BML-14, MGC-32 × BML-14, MGC-32 $\times$ GP-170, MGC-92 × GP-170, MGC-137 $\times$ GP-311, MGC-237 × BML-7, MGC-242 $\times$ BML-14, MGC-248 × GP-311, MGC-252 × BML-14, MGC-252 × GP-311, MGC-254 $\times$ BML-14, MGC-254 × GP-311, MGC-256 × GP-170 and MGC-256 $\times$ GP-311 were found resistant, 16 hybrids, viz., MGC- $6 \times$ BML-7, MGC-6 $\times$ BML-14, MGC-6 $\times$ GP-170, MGC$15 \times$ GP-311, MGC-32 × BML-6, MGC-32 $\times$ GP-311, MGC-137 × GP-170, MGC-230 $\times$ BML-7, MGC-230 × GP-311, MGC-238 $\times$ BML-7, MGC-239 $\times$ BML-14, MGC-239 $\times$ GP-311, MGC-242 $\times$ GP-170, MGC-248 $\times$ BML-14, MGC-252 × BML-6 and MGC-256 $\times$ BML-14 were moderately resistant, 22 hybrids, viz., MGC-6 × BML-6, MGC-6 $\times$ GP-311, MGC-9 $\times$ BML-7, MGC-9 $\times$ GP311, MGC-15 × BML-6, MGC-92 × BML-7, MGC-92 × GP-311, MGC-137 × BML-6, MGC-137 × BML-14, MGC-230 × BML-6, MGC-237 $\times$ GP-170, MGC-237 × GP-311, MGC-238 × BML-14, MGC-238 × GP-170, MGC-239 $\times$ BML-7, MGC-242 × BML-7, MGC-242 $\times$ GP-311, MGC-248 $\times$ BML-7, MGC-248 $\times$ GP-170, MGC-252 $\times$ GP-170, MGC-256 $\times$ BML-6 and MGC-256 $\times$ BML-7 were moderately susceptible, 22 hybrids, viz., MGC-9 × GP-170, MGC-15 × BML-7, MGC$15 \times$ BML-14, MGC-15 $\times$ GP-170, MGC-32 $\times$ BML-7, MGC-92 × BML-6, MGC-92 × BML-14, MGC-137 × BML-7, MGC-230 × BML-14, MGC-230 × GP-170, MGC-237 × BML-6, MGC-237 × BML-14, MGC-238 $\times$ BML-6, MGC-238 × GP-311, MGC-239 $\times$ BML-6, MGC-239 × GP-170, MGC-242 × BML-6, MGC-248 $\times$ BML-6, MGC-252 $\times$ BML-7, MGC-254 $\times$ BML-6, MGC-254 $\times$ BML-7 and MGC-254 $\times$ GP-170 were found susceptible.

The disease severity was recorded in the field by using a scale (1-9 cm) of Payak and Sharma (1983). All these maize inbred lines were screened in field by toothpick method of inoculation. As a result, most of the genotypes were exhibited disease reaction varying from resistant (score 2) to moderately resistant (score 5) against $M$. phaseolina. Disease severity data obtained from the field was summarized based on their average disease reaction presented in the (Table 4). In contrast, the currently followed inoculation procedure developed by Payak and Sharma (1983) requires a longer time of about 40 days for expression of plant drying symptoms due to PFSR and data are possible to record only at the time of crop harvesting.

In conclusion, the disease severity of PFSR is recorded in the field by observing the disease symptoms on the whole/individual plant. Hence, for recording data in field, Payak and Sharma (1983) prescribed 1-9 scale has been unequally distributed into four categories of disease severity, viz., resistant (1 to 3), moderately resistant (4 to 5), moderately susceptible (6 to 7) and susceptible reaction (8 to 9). This scale could be efficiently used for recording severity of PFSR in-vivo within 40 days following toothpick method of inoculations. The artificial epiphytotic condition for PFSR disease is created by inoculating the plants in the field just after flowering mainly by toothpick method of inoculation. But this method requires longer time for disease development and rotting symptoms in the inoculated stalks become prominent only at harvesting stage. In ordered to identified PFSR resistant lines, screening of 98 maize genotypes in field against $M$. phaseolina, only four lines, viz., MGC-237, MGC-248, MGC-254, MGC-256 and two testers, viz., BML-6 and GP-311. Whereas, 15 crosses viz., MGC-9 $\times$ BML-6, MGC-9 $\times$ BML-14, MGC-32 × BML-14, MGC-32 × GP-170, MGC-92 × GP-170, MGC-137 × GP-311, MGC-237 × BML-7, MGC-242 × BML-14, MGC-248 × GP-311, MGC-252 × BML-14, MGC-252 × GP-311, MGC-254 × 
BML-14, MGC-254 × GP-311, MGC-256 × GP-170 and MGC-256 $\times$ GP-311 were found resistant.

\section{Acknowledgement}

Authors are thankful to the Maize Research Centre, Agricultural Research Institute, Rajendranagar, Hyderabad for providing maize inbred lines.

\section{References}

AICRP, 2014. Annual Report of AICRP Maize Pathology Udaipur center.

Anonymous. 1983. The techniques of scoring for resistance to diseases of maize in India. All India Co-ordinated Maize Improvement Project, IARI, New Delhi, p133.

Anonymous. 2012. Inoculation Methods and Disease Rating Scales for Maize Diseases. Shekharm and Kumar Sangit (Eds). Directorate of Maize Research, ICAR, New Delhi.

Clark, R.L and Foley, D.C. 1985. Stalk rot resistance and strength of maize stalk from the plant introduction collection. Plant Disease 69: 419-22.

Desai, S., Hegde, R.K and Desai, S. 1991. A preliminary survey of incidence of stalk rot complex of maize in two districts of Karnataka. Indian Phytopathology 43: 575-6.

Harlapur, S.I., Wali, M.C., Prashan, $M$ and Shakuntala, N.M. 2002. Assessment of yield losses in maize due to charcoal rot in Ghataprabha Left Bank Canal (GLBC) command area of Karnataka. Karnataka
Journal of Agricultural Science 15: 590-1.

Hooda, K.S. 2012. Identifying sources of multiple disease resistance in maize. Maize Journal 1: 82-4.

India stat, 2016-17. https://www.indiastat.com/maize/productio n/area.

Kumar, M., Lal, H.C and Johan, M. 1998. Assessment of yield loss due to post flowering stalk rots in maize. Journal of Applied Biolology 8: 90-2.

Nagy, E and Cabulea, I. 1996. Breeding maize for tolerance to Fusarium stalk and ear rot stress. Romanian Agricultural Research No. 5(6): 45-52.

Payak, M.M and Sharma, R.C. 1983. Disease rating scales in maize in India. (In) Techniques of Scoring for Resistance to Diseases of Maize in India. All India Coordinated Maize Improvement Project, IARI, New Delhi, pp 1-4.

Payak, M.M and Sharma, R.C. 1985. Maize diseases and approaches to their management in India. Tropical Pest Management 31: 302-10.

Shankar Lingam, S and Venkatesh, S. 2005. New source of resistance to post Flowering Stalk rot in maize. Indian Journal of plant protection. 33(1): 99-101.

Sharma, R.C., Carlos De Leon and Payak, M.M. 1993. Diseases of maize in south and south- East Asia: Problems and Progress. Crop Protection. 12: 414-422.

Shekhar, M., Kumar, S., Sharma, R.C and Singh, R. 2010. Sources of resistance against post-flowering stalk rot of maize. Archives of Phytopathology and Plant Protection 43: 259-63

\section{How to cite this article:}

Banoth Madhu, K Prabhavathi, D Bhadru and Mallaiah, B 2021. Screening Technique for Identification of Resistant Genotypes against Post Flowering Stalk Rot Complex caused by Macrophomina phaseolina in Maize (Zea mays L.). Int.J.Curr.Microbiol.App.Sci. 10(01): 3535-3544. doi: https://doi.org/10.20546/ijcmas.2021.1001.417 